\title{
Frequency-Domain Analysis of A/D Converter Nonlinearity
}

\author{
Zhiqiang Gu† \\ Bell-Northern Research Ltd. \\ P.O.Box 3511, Station C \\ Ottawa, Ontario, Canada K1Y $4 \mathrm{H} 7$ \\ Email:ggu@bnr.ca \\ W. Martin Snelgrove† \\ Dept. of Electronics \\ Carleton University \\ Ottawa, Ontario, Canada K1S 5B6 \\ Email: snelgar@keiko.doe.carleton.ca
}

†Previously with Dept. of Electrical and Computer Engineering, University of Toronto,
Toronto, Ontario, Canada M5S 1A4

TOPIC: CIRCUITS AND SYSTEM THEORY OR ANALOG CIRCUITS AND FILTERS

\begin{abstract}
A novel two-dimensional z-transform approach to the analysis of analog/digital $(A / D)$ converter nonlinearity is presented. It was primarily developed to study the stability behavior of an adaptive self-trimming technique for flash and multi-stage $A / D$ converters, but has more general applicability.
\end{abstract}

\section{INTRODUCTION}

An adaptive self-trimming algorithm for $A / D$ converters $[1,2]$ has been presented which continually trims thresholds in the flash $A / D$ subconverters of two-stage and pipelined $A / D$ converters. Roughly speaking, it trims thresholds up or down by small increments in such a way as to smooth out irregularities in the code density of the A/D subconverters' digital output. To demonstrate its stability and predict convergence rate, we developed a novel two-dimensional (2-D) z-transform approach. This paper introduces the 2-D z-transform approach, and illustrates its general applicability in frequency-domain interpretation of the distinct properties of differential and integral nonlinearity in $A / D$ converters and its applicability in describing a simple adaptive threshold-trimming system. The application of this approach for a comprehensive analysis of the practical adaptive self-trimming systems presented in [1,2] is presented in [3].

Figure 1 shows the input-output characteristic of an ideal 2-bit flash analog/digital converter. It is like a staircase, with integer output values and "risers" at threshold values $x_{i}$, which are ideally 
spaced uniformly one "least-significant bit (LSB)" $\Delta$ apart. Mathematically, these ideal thresholds are $\hat{x}_{i}=i \Delta$, and the converter's output is $i=\left\lfloor v_{i n} / \Delta\right\rfloor$. Several definitions are possible, some allowing negative outputs and most with the staircase shifted $\Delta / 2$ vertically or horizontally from that shown in Figure 1, but we have chosen this one because it simplifies the analysis and the extension to the others is routine.

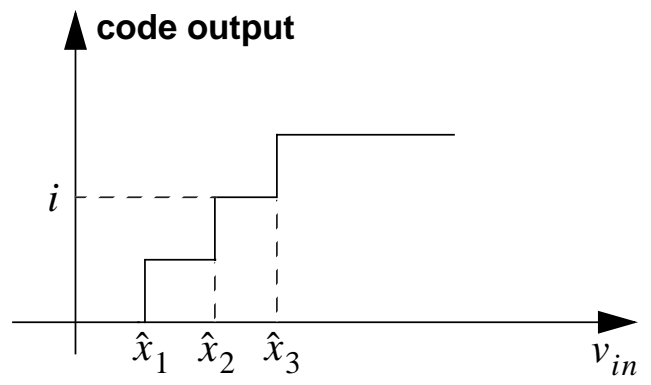

\section{Fig. 1 Input/output characteristic of an ideal 2-bit A/D converter}

A practical flash $A / D$ converter has its thresholds randomly shifted from the ideal thresholds $\hat{x}_{i}$, resulting in A/D converter nonlinearity. Two measures commonly used are differential nonlinearity (DNL), which tends to emphasize fine-scale errors, and integral nonlinearity (INL), which shows up cumulative errors affecting the overall shape of the characteristic function.

Mathematically, a practical flash A/D converter produces a digital output

$$
D(\mu)=i, \mu \in\left[x_{i}, x_{i+1}\right)
$$

DNL is defined as

$$
D N L_{i}=x_{i+1}-x_{i}-\Delta
$$

and INL is deviation of a threshold $x_{i}$ from its ideal case $\hat{x}_{i}$, which can also be written as a running sum (hence the word "integral") of DNL 


$$
I N L_{i}=x_{i}-i \Delta=\sum_{j=0}^{i} D N L_{j}
$$

An N-bit flash A/D converter only define $2^{N}-1$ thresholds, $x_{1}$ up to $x_{2^{N}-1}$. End-points $x_{0} \equiv 0$ and $x_{2^{N}} \equiv 2^{N} \Delta$ are defined so that an ideal converter has zero INL and DNL everywhere. We will be paying a lot of attention to this function in the rest of this paper, so we'll give it the name $\gamma_{i}=I N L_{i}$ for notational convenience (accordingly, $\hat{\gamma}_{i}$ as its ideal case). As an example, Figure 2 shows the input-output characteristic of a 2-bit flash AID converter with a $0.5 \Delta$ threshold error in $x_{2}$. Its INL has $\gamma_{2}=0.5$ and is otherwise zero.

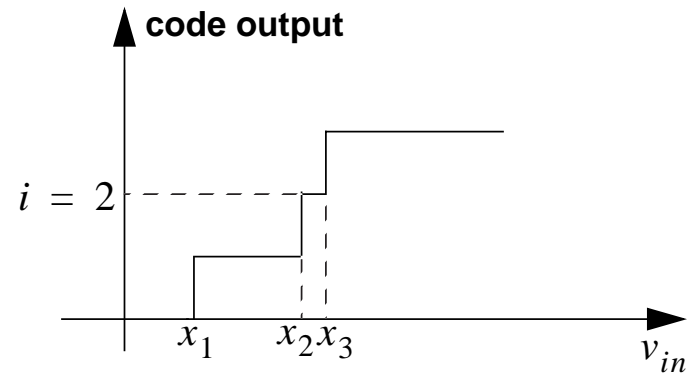

\section{Fig.2 Characteristic of an A/D converter with a threshold error}

\section{Z-TRANSFORM IN THE CODE DOMAIN}

The set of $A / D$ converter thresholds $x_{i}$ can be regarded as a signal sequence, and then described

by a generic z-transform [4]. Generally,

$$
Z\left\{x_{i}\right\}=\sum_{i=1} x_{i} z^{-i}
$$

For example, the ideal 2-bit flash AID converter with a set of thresholds $\left\{x_{1}, x_{2}, x_{3}\right\}=\{1,2,3\}$ has $X(z)=z^{-1}+2 z^{-2}+3 z^{-3}$. The coefficients of the equation 
have a "ramp" form, so there is an equivalent form with a denominator having a double pole at $z=1:$

$$
X(z)=\frac{1}{\left(1-z^{-1}\right)^{2}}\left(z^{-1}-4 z^{-4}+3 z^{-5}\right)
$$

The numerator in Eq.(5) contains large terms in $z^{-4}$ and $z^{-5}$ that come from having finite $N$. The z-transform of INL is more convenient: ideally it's $\Gamma(z)=Z\left\{\hat{\gamma}_{i} \equiv 0\right\}=0$, and in practice it's a polynomial of order $2^{N}-1$ in $z^{-1}$ with small coefficients corresponding to individual threshold errors. DNL is just the "derivative" of INL

$$
Z\left\{D N L_{i}\right\}=(z-1) \Gamma(z)
$$

For the example shown in Figure 2, $Z\left\{D N L_{i}\right\}=0.5 z^{-1}-0.5 z^{-2}$

The important point of using such a z-transform is that it gives us a definition of "frequency", with which we can distinguish between "high-frequency" errors (which model local variation and dominate quantization noise performance) and "low-frequency" errors (which model variations of the overall characteristic from linearity and which dominate low-order harmonic distortion). Evaluating the z-transform of INL at an angle $\theta$ on the unit circle gives us

$$
\Gamma\left(e^{j \theta}\right)=\sum_{i=1}^{2^{N}-1} \gamma_{i} e^{i j \theta}
$$

which correlates errors with sinusoids in code $i$. For example, at $\theta=(2 \pi) /\left(2^{N}-1\right)$ the real and imaginary parts of $e^{i j \theta}$ run through a single cycle each of $\cos$ and $\sin$, and a large $\operatorname{Im} \Gamma\left(e^{j \theta}\right)$ shows that the overall characteristic has a shape like that shown in Figure 3. This would produce significant third-order inter-modulation distortion when converting a large signal.

Notice that, by differentiating Eq.(7), we can derive the z-transformed DNL, and DNL naturally 
emphasizes its high-frequency components

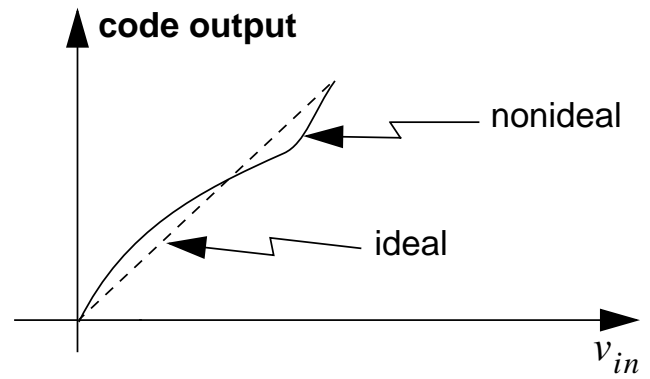

\section{Fig.3 Characteristic of an A/D converter with a low-frequency threshold error}

\section{2-D z-TRANSFORM TO DESCRIBE TRIMMING ALGORITHM}

We are ultimately interested in error-correction algorithms that trim flash AID thresholds during operation, so that the thresholds $x_{i}$ become functions of time, i.e., $x_{i}(t)$. Expressing time variable $t$ in terms of sample number $k$, we want to understand the behavior of $x_{i}(k)$. We can then use z-transform techniques on these threshold variables to see whether a trim algorithm can drive INL and DNL to zero, and how quickly.

Thresholds $x_{i}$ are now functions of two variables, output code $i$ and time $k$. Thus we need a twodimensional z-transform to describe them. As before, it is convenient to focus on $\operatorname{INL} \gamma_{i}(k)$ :

$$
\Gamma\left(z_{c}, z_{t}\right)=Z_{c, t}\left\{\gamma_{i}(k)\right\}=\sum_{k=0}^{\infty} \sum_{i=1}^{2^{N}-1} \gamma_{i}(k) z_{c}^{-i} z_{t}^{-k}
$$

where the two variables $z_{c}$ and $z_{t}$ transform the time and code domains respectively. For example, if there is a simple offset error that drops geometrically to zero with time, thresholds will be $x_{i}=i \Delta+\varepsilon^{k}$ and errors $\gamma_{i}(k)=\varepsilon^{k}$. The z-transform of the errors $\gamma_{i}(k)$ is 


$$
\Gamma\left(z_{c}, z_{t}\right)=\left(z_{c}^{-1}+z_{c}^{-2}+\ldots+z_{c}^{-\left(2^{N}-1\right)}\right)\left(1+\varepsilon z_{t}^{-1}+\varepsilon^{2} z_{t}^{-2}+\ldots\right)
$$

and the term in $z_{t}$ can be rewritten as $1 /\left(1-\varepsilon z_{t}^{-1}\right) \quad($ as long as $|\varepsilon|<1)$.

Now the final-value theorem [4] tells us that a trimming algorithm can eventually drive errors to

$$
\lim _{z_{t} \rightarrow 1}\left(z_{t}-1\right) \Gamma\left(z_{c}, z_{t}\right)
$$

and, for the trivial case of Eq.(9), the final error is zero. In general, unfortunately, two-dimensional transforms will not have the convenient separable form of Eq.(9), but we will still be able to make use of them (see beflow and [3]).

Let us now look at a more useful example in which a trim algorithm adaptively drives flash $A / D$ thresholds towards mean of neighbors. The trim algorithm can be mathematically expressed as:

$$
x_{i}(k)=x_{i}(k-1)+\mu\left[x_{i+1}(k-1)-2 x_{i}(k-1)+x_{i-1}(k-1)\right], \text { at time } k T
$$

with two fixed end-points at: $x_{0}(k) \equiv 0$ and $x_{2^{N}}(k) \equiv V_{\text {ref }}$ for all $k$, where $\mu$ is a small-value adaptation step size. For simplicity, we use a 2-bit flash A/D converter as our illustrative example, for which the threshold sequence $\hat{x}_{i}(k)$ in the ideal case can be written as:

$$
\hat{x}_{i}(k)=0,1,2,3,4 L S B
$$

A set of state-space equations for this adaptive 2-D system can thus be written in matrix form as:

$$
\left[\begin{array}{c}
x_{1}(k+1) \\
x_{2}(k+1) \\
x_{3}(k+1)
\end{array}\right]=\left[\begin{array}{ccc}
1-2 \mu & \mu & 0 \\
0 & 1-2 \mu & \mu \\
0 & \mu & 1-2 \mu
\end{array}\right]\left[\begin{array}{l}
x_{1}(k) \\
x_{2}(k) \\
x_{3}(k)
\end{array}\right]+\left[\begin{array}{c}
0 \\
0 \\
4 \mu
\end{array}\right]
$$

or $\dot{X}=A X+B$. We can examine the stability of this system by evaluating the eigenvalues of the 
characteristic function $\operatorname{det}[\lambda I-A]=0$. The eigenvalues of this function can be readily found as:

$$
\begin{aligned}
& \lambda_{1}=1-2 \eta \\
& \lambda_{2,3}=1-2 \eta \pm \sqrt{2} \eta, \text { where } \eta>0
\end{aligned}
$$

The eigenvalues are actually the poles of the state-space system in the $z_{t}$ plane. From Eq. (13), we can see that regardless of the value of $\eta$, these poles are always all located inside the unit circle, i.e., the stability of this system is guaranteed. When $\eta=0.1$, for example, we have $\lambda_{1}=0.8, \lambda_{2}=0.9414$, and $\lambda_{3}=0.6586$. The conclusion from such a 2-bit flash A/D converter applies to a larger system, since the larger system has a larger matrix $A$ but the regular structure of the matrix remains the same

This adaptive 2-D system can be readily analyzed by the 2-D z-transform. As discussed above, we focus on INL $\gamma_{i}(k)$ which gives our favoured boundary condition: $\gamma_{i}(k) \equiv 0$ for $i \notin\left[1,2^{N}-1\right]$. In terms of $\gamma_{i}(k)$, Eq.(11) can be rewritten as:

$$
\gamma_{i}(k)=\gamma_{i}(k-1)+\mu\left[\gamma_{i+1}(k-1)-2 \gamma_{i}(k-1)+\gamma_{i-1}(k-1)\right]
$$

The 2-D z-transform of Eq.(14) is

$$
\begin{gathered}
\Gamma\left(z_{c}, z_{t}\right)=\frac{z_{t}}{z_{t}-1} Z\left\{\gamma_{i}(0)\right\}+\frac{\mu}{z_{t}-1}\left(z_{c}-2+z_{c}^{-1}\right) \Gamma\left(z_{c}, z_{t}\right) \\
\text { where } \Gamma\left(z_{c}, z_{t}\right)=Z\left\{\gamma_{i}(k)\right\}
\end{gathered}
$$

and then

$$
H\left(z_{c}, z_{t}\right)=\frac{\Gamma\left(z_{c}, z_{t}\right)}{Z\left\{\gamma_{i}(0)\right\}}=\frac{z_{t}}{z_{t}-1-\mu\left(z_{c}-2+z_{c}^{-1}\right)}
$$

The transfer function of the 2-D system is not generally separable in terms of $z_{c}$ and $z_{t}$, but for our 
A/D converter case this doesn't complicate our analysis much because both the adaptation step size $\mu$ and the term $\left(z_{c}-2+z_{c}^{-1}\right)$ in the denominator of Eq.(15B) (the latter term reflects the deviation of $z_{c}$ values from their ideal cases) are always relatively small compared to unity. In this case we can see from Eq.(15B) that the 2-D system behaves as a low-pass (LP) filter in the $z_{t}$ domain. In the equation, the output $\Gamma\left(z_{c}, z_{t}\right)$ is the $i$-th threshold at time $k$, and the input $Z\left\{\gamma_{i}(0)\right\}$ the initial value of the $i$-th threshold which exists prior to the adaptation process. The second term of Eq.(15A) represents the threshold nonuniformity of the threshold array, because $\left(z_{c}-2+z_{c}^{-1}\right)=\left(z_{c}-1\right)^{2} z_{c}^{-1}$ estimates the second derivative. When all three thresholds of the 2-bit A/D converter are perfectly aligned, this term should perish. Otherwise, the second term is expected to compensate the error portion of the initial threshold $\gamma_{i}(0)$.

The "first"-order LP filter has multiple poles (from the variable $z_{c}$ ) in the $z_{t}$ plane at:

$$
z_{t}=1+\mu\left(z_{c}-2+z_{c}^{-1}\right)
$$

The $z_{c}$ values at equilibrium from the 2 -bit $\mathrm{A} / \mathrm{D}$ converter can be estimated by

$$
\begin{aligned}
Z\left\{\hat{\gamma}_{i}\right\} & =\sum_{i=1}^{3} \hat{A} z_{c}^{-i}, \text { where } \hat{A} \rightarrow 0 \\
& =\hat{A} \cdot z_{c}^{-3}\left(z_{c}^{2}+z_{c}+1\right)
\end{aligned}
$$

which has the following two ideal zeros:

$$
\hat{z}_{c(1,2)}=e^{j \frac{2 \pi}{3}}, e^{j \frac{4 \pi}{3}}
$$

These zeros are all on the unit circle, as shown in Figure 4 (other detail of the figure is to be explained below). 


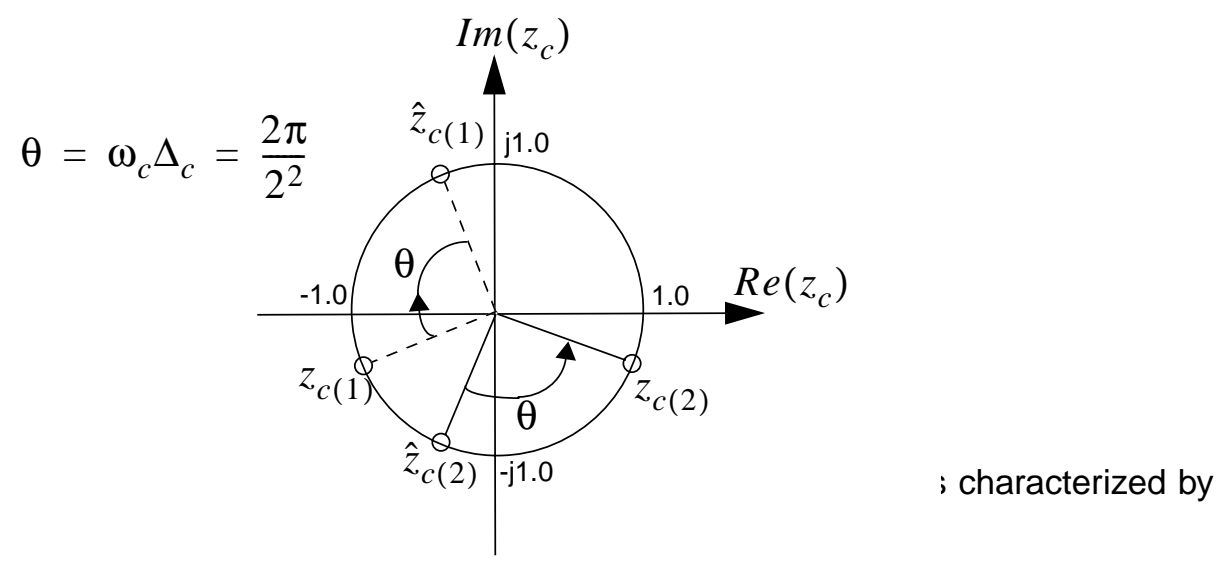

INL:

$$
\gamma_{i}=\quad \text { Fig.4 Zero location of an INL sequence }
$$

$$
\text { the "sampling" interval } \Delta_{c}=1 L S B
$$

We can define the angular frequency $\omega_{c}$ in Eq.(19) as $\omega_{c}=2 \pi \frac{M}{4 \Delta_{c}}$ (unit of $\frac{\omega_{c}}{2 \pi}=1 /$ volt) where $M$ is the number of the periods of the INL sequence $\gamma_{i}$. Figure 5 shows the variation of the threshold sequence $x_{i}$ from its ideal case $\hat{x}_{i}\left(\right.$ recall that $\gamma_{i}=x_{i}-\hat{x}_{i}$ ) when $\alpha=1$ and $M=1$, i.e., a low-frequency distortion occurs. In this case we have the z-transform of the INL's periodic complex exponential $\gamma_{i}=A e^{j \omega_{c} \Delta_{c} i}$ 


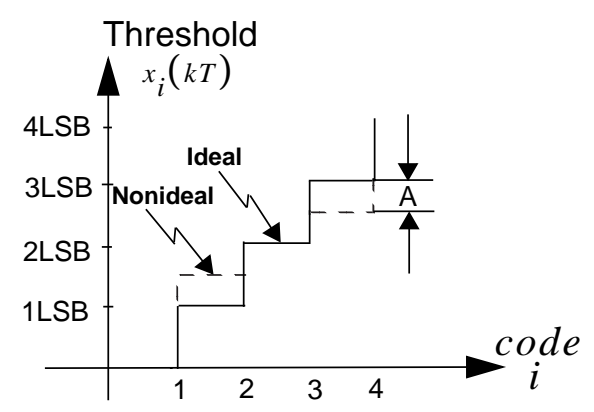

(a) Transfer curve of a 2-bit A/D converter

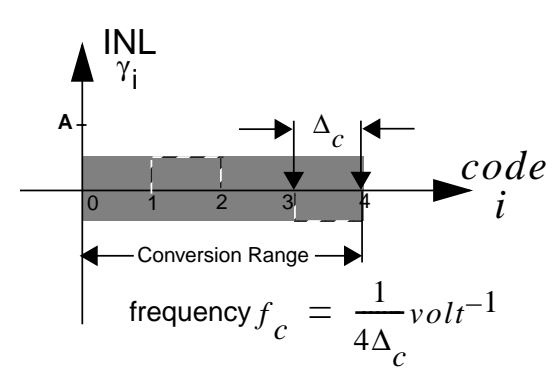

(b) Corresponding INL

Fig.5 Integral nonlinearity of a threshold sequence

$$
\begin{aligned}
Z\left\{\gamma_{i}\right\} & =\sum_{i=1}^{3} A e^{j \omega_{c} \Delta_{c} i} z_{c}^{-i} \\
& =A \cdot R \cdot z_{c}^{-3}\left(z_{c}^{2}+R z_{c}+R^{2}\right), \text { where } R=e^{j \omega_{c} \Delta_{c}}
\end{aligned}
$$

Two offset zeros for this threshold sequence can be found from Eq.(20):

$$
z_{c 0(1,2)}=R e^{j \frac{2 \pi}{3}}, R e^{j \frac{4 \pi}{3}}
$$

which are shown together with the two ideal zeros in Figure 4. The filtering effect of our adaptive trimming technique is to move these offset zeros $z_{c 0(1)}$ and $z_{c 0(2)}$ to their ideal positions by an angle of $\theta=\omega_{c} \Delta_{c}=\frac{2 \pi}{2^{2}}$. 
In [3], we apply the 2-D z-transform to analyze a practical histogram-based threshold-trimming system which consists of the core of an on-line self-calibration technique for multi-stage A/D converters presented in $[1,2]$

\section{CONCLUSION}

A novel two-dimensional z-transform approach to the analysis of $A / D$ converter nonlinearity has been presented. The point of using such an approach is that it gives us a definition of "frequency", with which we can distinguish between errors resulting from the local variations of an $A / D$ transfer curve and those from the variation of overall characteristic from linearity. It has been found that integral nonlinearity (INL) is more convenient to use in the frequency-domain analysis. By trimming $A / D$ converter thresholds in some way such as in a simple state-space system illustrated in this paper and those practical systems in $[1,2]$, we can eventually drive integral nonlinearity and differential nonlinearity to zero. The 2-D z-transform can be applied to reveal the functionality and stability behavior of these systems.

\section{REFERENCES}

[1]. Z. Gu and W.M.Snelgrove, "A novel self-calibrating scheme for video-rate 2-step A/D converter", Proceedings of IEEE 1992 International Symposium on Circuits and Systems, Vol.2, pp601-604, San Diego, May 1992.

[2]. Z. Gu and W. M. Snelgrove, "Application of a novel self-calibrating technique to pipelined multistage A/D converter", Proceedings of 35th Midwest Symposium on Circuits and Systems, Vol.1, pp64-67, Washington, DC., Aug.1992.

[3]. Z. Gu and W. M. Snelgrove, "Analysis and Design of Adaptive Self-Trimming Algorithm for A/D Converters", submitted IEEE 1994 International Symposium on Circuits and Systems, London, England, May 1994.

[4]. A. V. Oppenheim, A. S. Willsky and I. T. Young, "Signals and Systems", Prentice-Hall, 796pp, 1983. 